\title{
The XMM-Newton view of stellar coronae: $X$-ray spectroscopy of the corona of AB Doradus ${ }^{\star}$
}

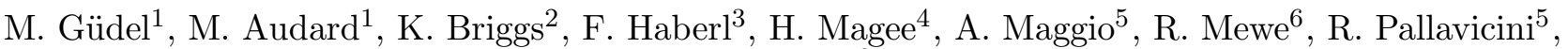 \\ and J. Pye ${ }^{2}$ \\ 1 Paul Scherrer Institut, Würenlingen \& Villigen, 5232 Villigen PSI, Switzerland \\ 2 Dept. of Physics \& Astronomy, University of Leicester, University Road, Leicester LE1 7RH, UK \\ 3 Max-Planck-Institut für Extraterrestrische Physik, PO Box 1312, 85741 Garching, Germany \\ 4 Mullard Space Science Laboratory, University College London, Holmbury St. Mary, Dorking, \\ Surrey RH5 6NT, UK \\ 5 Osservatorio Astronomico di Palermo "G. S. Vaiana", Piazza del Parlamento 1, 90134 Palermo, Italy \\ 6 SRON Laboratory for Space Research, Sorbonnelaan 2, 3584 CA Utrecht, The Netherlands
}

Received 2 October 2000 / Accepted 8 November 2000

\begin{abstract}
We report results of deep X-ray observations of AB Doradus obtained with the XMM-Newton observatory during its Performance Verification phase. The main objective of the analysis is a study of the spectral variability of coronal plasma in a very active star, including investigations of the variable thermal structure, abundance variations, and possible density changes during flares. AB Dor revealed both quiescent and flaring emission. The RGS spectra show flux changes in lines of highly ionized Fe during the flares, and an increase of the continuum. Elemental abundances increase in the early flare phases, by a factor of three. The quiescent abundances are lower than corresponding solar photospheric values, and tend to increase with increasing first ionization potential, contrary to the behavior in the solar corona. High-resolution spectra show an average density of the cool plasma of $\approx 310^{10} \mathrm{~cm}^{-3}$; this value does not change during the flares. We analyse and model the temporal behavior of heating and cooling, and present model results for one of the flares. We find that magnetic loops with a semilength of the order of $2.510^{10} \mathrm{~cm} \approx 0.3 R_{\star}$ are involved.
\end{abstract}

Key words. stars: abundances - stars: activity - stars: coronae - stars: flare - stars: individual: AB Doradus X-rays: stars

\section{Introduction}

Late-type stars that have newly arrived on the Zero-Age Main-Sequence (ZAMS) are ideal objects for coronal studies since they are supposed to lack strong circumstellar disks while showing an enhanced level of activity, due to their high rotation rates. AB Doradus, first detected in X-rays by Pakull (1981), is a particularly interesting nearby example. Initially identified as a pre-main sequence weak-lined T Tau star, it is now believed to be located precisely on the ZAMS, based on accurate distance information from Hipparcos (ESA 1997; $14.9 \pm 0.1 \mathrm{pc})$ and VLBI (Guirado et al. 1997; $15.0 \pm 0.1 \mathrm{pc}$ ). Its age has been estimated to be 20-30 Myr (Collier Cameron \& Foing 1997). Its short rotation period of $0.514 \mathrm{~d}$ (Pakull 1981) is

Send offprint requests to: M. Güdel; e-mail: guedel@astro.phys.ethz.ch

* Based on observations obtained with XMM-Newton, an ESA science mission with instruments and contributions directly funded by ESA Member States and the USA (NASA). thought to be responsible for the very high ("saturated") X-ray luminosity of $\approx 10^{30} \mathrm{ergs} \mathrm{s}^{-1}$, with $L_{\mathrm{X}} / L_{\mathrm{bol}} \approx 10^{-3}$ (Vilhu \& Linsky 1987). AB Dor has been a favorite object for the study of stellar flares, showing an X-ray flare rate of $\approx$ two per day (Vilhu et al. 1993). For further overall properties of AB Dor, we refer to Maggio et al. (2000).

Being the nearest K-type ZAMS star, AB Dor has been the subject of extensive investigations across all wavelength bands. X-ray observations were discussed by Vilhu \& Linsky (1987), Collier Cameron et al. (1988), Vilhu et al. (1993), Rucinski et al. (1995), Mewe et al. (1996), White et al. (1996), Kürster et al. (1997), Ortolani et al. (1998), and Maggio et al. (2000). High activity levels were consistently reported, with extremely powerful flares reaching temperatures (T) of 100 MK (Maggio et al. 2000); unusually low coronal abundances were reported from $A S C A$, BeppoSAX, and EUVE, the typical metallicity level being at $Z \approx 0.3 Z_{\odot}$ (Mewe et al. 1996; Ortolani et al. 1998; Maggio et al. 2000) despite the measured solar-like photospheric abundances of this star (Vilhu et al. 1987). 
Table 1. Observing log

\begin{tabular}{llc}
\hline & First observation: & 2000 April 30/May 1 \\
\hline Instrument & UT range & JD range 2451665.0 + \\
\hline RGS & $19: 19: 12-00: 29: 24$ & $0.30500-0.52042$ \\
& $02: 30: 21-19: 46: 29$ & $0.60441-1.32395$ \\
pn & $19: 49: 42-23: 27: 54$ & $0.32618-0.47771$ \\
\multirow{2}{*}{ MOS1 } & $02: 30: 21-19: 32: 29$ & $0.60441-1.31422$ \\
MOS2 & $22: 02: 17-00: 07: 09$ & $0.41825-0.50497$ \\
\hline \multirow{2}{*}{ Instrument } & UT range & JD range $2451702.0+$ \\
\hline RGS & $05: 29: 46-21: 53: 16$ & $0.72900-1.41199$ \\
pn & $09: 44: 12-21: 22: 32$ & $0.90569-1.39065$ \\
\hline
\end{tabular}

AB Dor is an ideal object to study spectral variability in active stellar coronae. X-ray spectroscopy is well suited to investigate the thermal structure, the abundance stratification, and densities of its corona. For this purpose, we have obtained long observations of AB Dor with the XMM-Newton X-ray observatory. The telescope's unrivaled sensitivity combined with AB Dor's high X-ray flux allow us to study time variability of the above diagnostics. The present Letter discusses first results from this campaign.

\section{Observations and data analysis}

The observations reported here were obtained by $X M M$ Newton (Jansen et al. 2001) in two closely spaced intervals on 2000 April 30/May 1, and on 2000 June 7 (Table 1). We refer to den Herder et al. (2001), Strüder et al. (2001), and Turner et al. (2001) for details on the X-ray instruments. In short, three telescopes focus X-rays onto three EPIC cameras (two MOS and one pn camera, sensitivity range $0.1-15 \mathrm{keV})$. About half of the photons in the converging beams of the telescopes that feed the MOS instruments are diffracted by sets of reflection gratings, and are then focused onto the RGS detectors. The RGS spectrometers provide spectral resolution of $\approx 70-500$ from $5-35 \AA$ $(0.35-2.5 \mathrm{keV})$. The EPIC pn observed in the small window mode, while the EPIC MOS's used the full window mode in the first observation but were closed during the later observations. MOS1\&2 saw only a few hours of exposure time in total, and will not be further discussed. With regard to EPIC pn, we concentrate on the first observation as this is the only observation to date that has been processed with the small window calibration. It is also the most interesting observation to study spectral variability.

All data were reduced with the XMM-Newton Science Analysis System (SAS) software v4.1, using several updates of individual tasks. For the RGS data, standard processing was performed using the RGSPROC task, followed by the spectral extraction and response generation. Analogously, we reduced the pn data with the
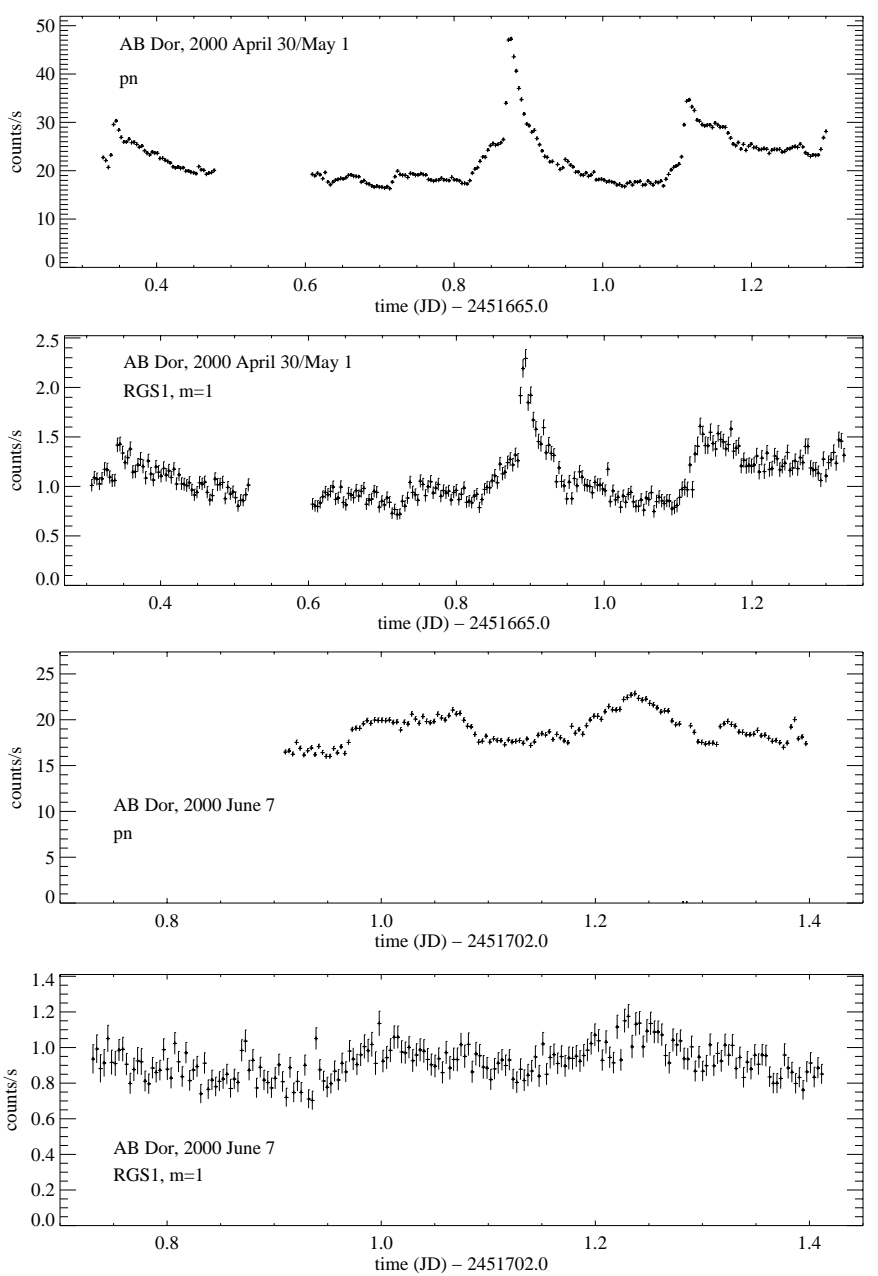

Fig. 1. Light curve of AB Dor derived from EPIC pn and RGS1, for the April 30/May 1 observations (upper two panels), and for the June 7 observation (lower two panels). Bin size is $300 \mathrm{~s}$ in each plot

EPPROC task. The spectral products were analysed in the Utrecht spectral software SPEX v.2.0 using the MEKAL Collisional Ionization Equilibrium model (Kaastra et al. 1996), and in XSPEC v11.0 (Arnaud 1996) using the corresponding VMEKAL model. Although the calibration of the response of both RGS is expected to evolve over time, we have used RGS2 for the spectral fit analysis as its calibration is more advanced than for RGS1, except for the analysis of the $\mathrm{O}$ VII triplet that is not available from RGS2 (due to the loss of one CCD, see den Herder et al. 2001).

The two observations show a very different behavior (Fig. 1). While the first observation reveals repeated flaring, the June observation is dominated by quiescent and slowly varying emission. No evidence for rotational modulation is seen. To identify heating events for further modeling, we defined a hardness ratio (HR) for pn spectra as $(B-A) /(A+B)$, where $A$ is the number of counts in the $0.3-1.0 \mathrm{keV}$ band and $B$ the number of counts in the $1.0-10 \mathrm{keV}$ band. This ratio was found to be sensitive to $T$ variations in a single- $T$ MEKAL plasma, while $A$ 


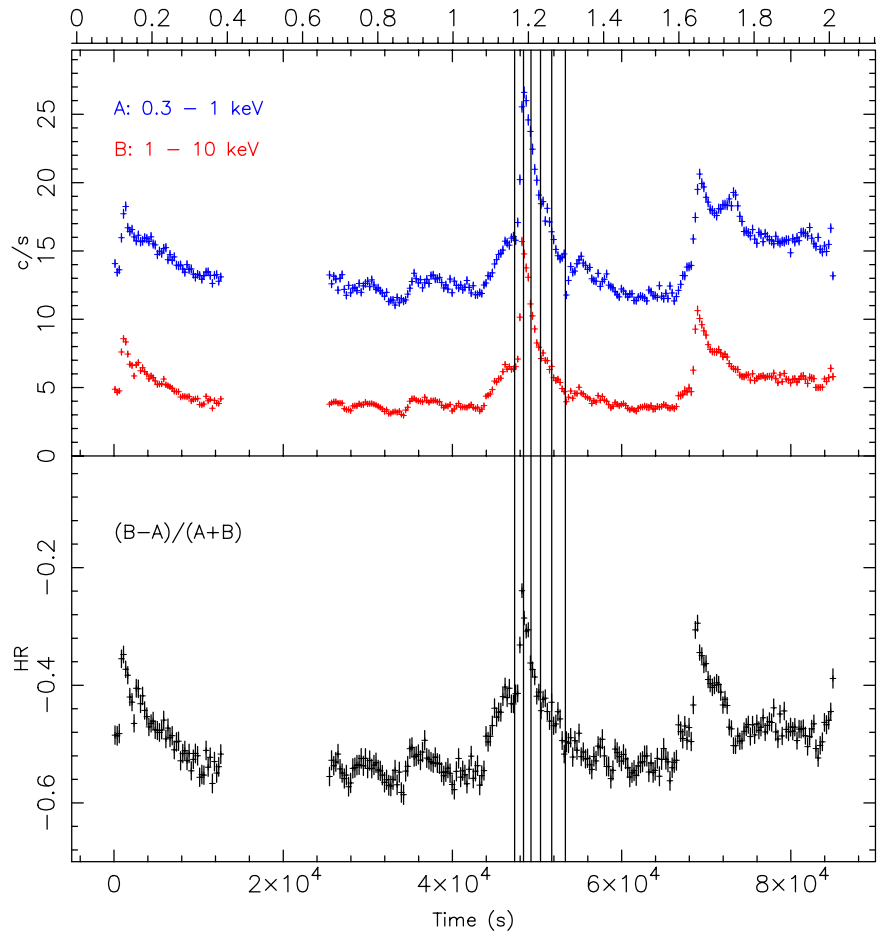

Fig. 2. EPIC pn light curves of $\mathrm{AB}$ Dor in the $0.3-1.0(A)$ and $1.0-10 \mathrm{keV}(B)$ bands and hardness ratio $(B-A) /(A+B)$. Only the first observation is shown. The topmost scale shows the rotational phase calculated from the ephemeris of Innis et al. (1988). The vertical lines define the time segments discussed in Sect. 4

and $B$ contain a comparable number of counts to minimise the uncertainty in HR. Background-subtracted light curves in $A$ and $B$ were constructed from counts in the source region ( $27^{\prime \prime}$ radius) and a distant background region (Fig. 2, top). The light curves again show the three flares and some additional modulation. A plot of the HR with time (Fig. 2, bottom) clearly indicates a correlation between count-rate and hardness (and hence plasma $T$ ).

Figures 3a,b show the coadded AB Dor high-resolution RGS spectra, calibrated in flux, during quiescence and during the two larger flares (at $0.9 \mathrm{~d}$ and $1.1 \mathrm{~d}$, total of $\approx 8 \mathrm{ks}$ ). A series of highly ionized Fe lines and several lines of the Ly and He-like series are visible, most notably from $\mathrm{C}, \mathrm{N}, \mathrm{O}, \mathrm{Ne}, \mathrm{Mg}$, and $\mathrm{Si}$. During the flares, strong additional lines appear around 10-12 $\AA$ which are partly attributed to Fe XXIV (see difference spectrum in Fig. 3c). There is also a significant change in the continuum during the flare (excess continuum suggested by the "ratio" plot in Fig. 3d). Both changes suggest a significantly increasing emission measure $(E M)$ at very high temperatures during the flares. Details on the time-variable thermal structure and on variable elemental abundances will be discussed in Sect. 4.

\section{Densities from the RGS}

The RGS range contains the density-sensitive line triplets of N vi, O viI, Ne IX, Mg XI, and Si XIII. Of these, only the

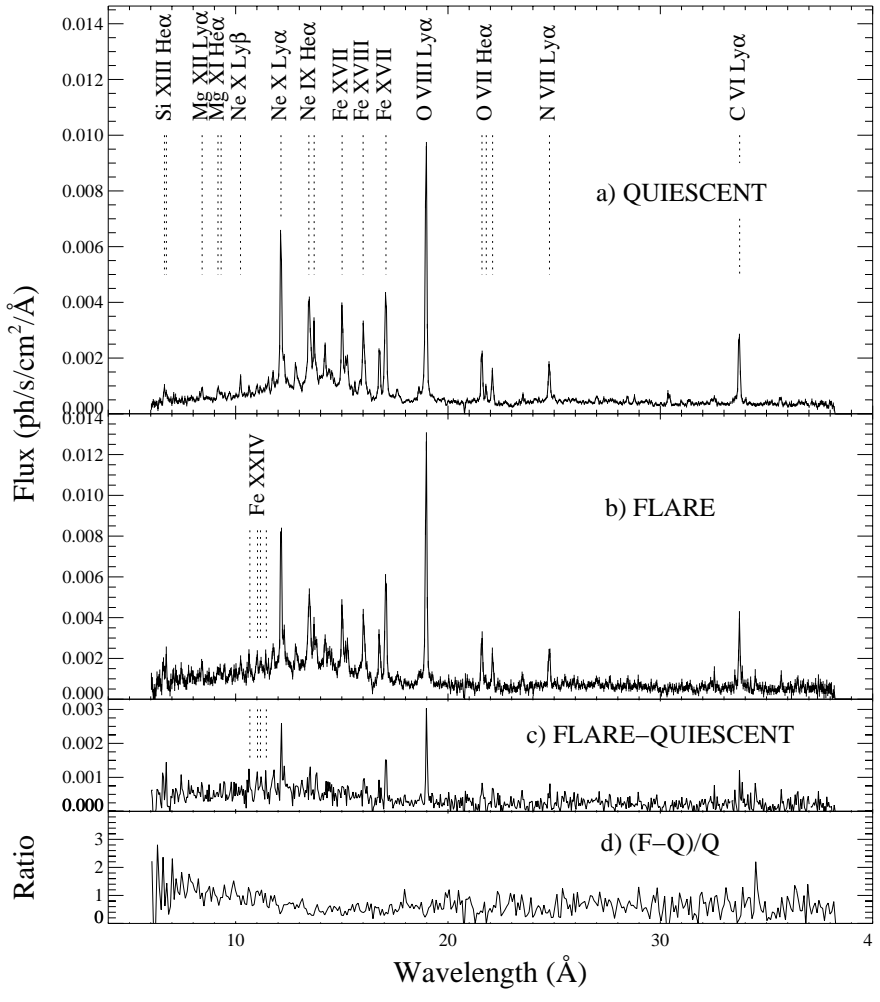

Fig. 3. RGS fluxed spectrum of the a) quiescent and b) flaring $\mathrm{AB}$ Dor, c) the difference spectrum, and $\mathbf{d}$ ) the ratio "(flare-quiescent)/quiescent". Data are binned to a resolution of $0.04375 \AA$ for a-c) and to $0.0875 \AA$ for d). Note different flux scales. The Fe XxIV lines and an excess continuum shortward of $10 \AA$ are evident in c) and d)

O viI triplet at $22 \AA$, formed at $1-3 \mathrm{MK}$, is strong enough in $\mathrm{AB}$ Dor for further analysis. We derived an electron density of $n_{\mathrm{e}}=(3 \pm 1.5) 10^{10} \mathrm{~cm}^{-3}$ from a global fit in SPEX (90\% confidence intervals for a single parameter of interest, $\left.\Delta \chi^{2}=2.71\right)$ - see Fig. 4. Combining data from the two larger flare peaks (3000 s around flare peak at $0.9 \mathrm{~d}$, and $5000 \mathrm{~s}$ around peak at $1.1 \mathrm{~d}$ ) we found $n_{\mathrm{e}}<$ $510^{10} \mathrm{~cm}^{-3}$, comparable with the quiescent value, i.e., there is no appreciable change in the average density of the emitting cooler material.

To confirm these results, we used an alternative approach by fitting the calibrated line spread function to the individual forbidden $(f)$ and intercombination $(i)$ lines. A flux ratio $f / i=2.34 \pm 0.69$ was found. Given that the emission is most probably originating from plasma with $T \approx 2-3 \mathrm{MK}$ (Sect. 4.1), the new calculations by Mewe et al. (2001) for the O VII triplet indicate $n_{\mathrm{e}}=\left(2.1_{-1.3}^{+2.3}\right) 10^{10} \mathrm{~cm}^{-3}$ for $T=3 \mathrm{MK}$, and $n_{\mathrm{e}}=\left(2.5_{-1.4}^{+2.7}\right) \quad 10^{10} \mathrm{~cm}^{-3}$ for $T=2 \mathrm{MK}$. Both values are in very good agreement with the SPEX global fit result.

\section{Time-resolved spectroscopy}

We examined the temperature variation and possible changes in the elemental abundances by performing 

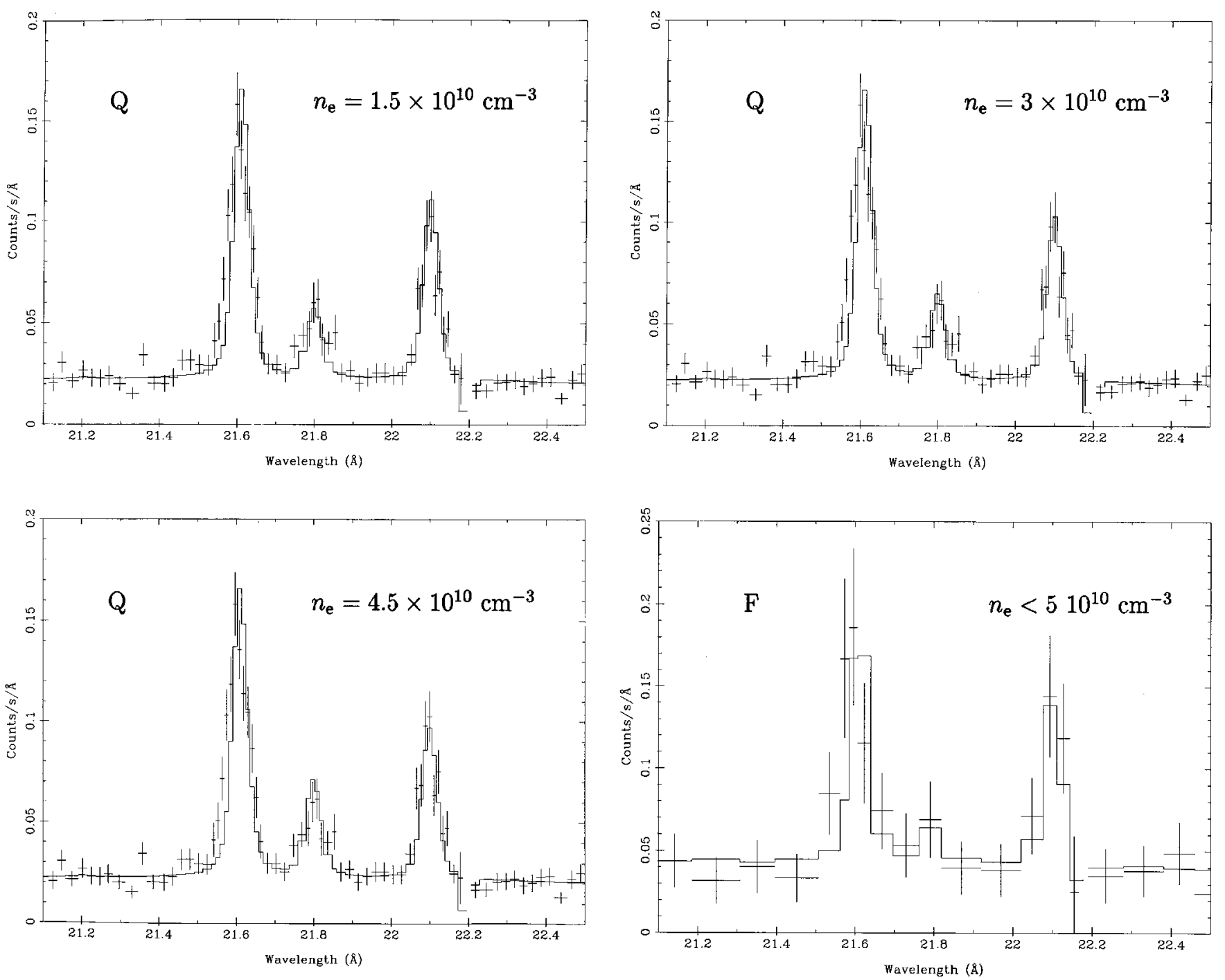

Fig. 4. Density-sensitive line triplet of He-like O viI (resonance, intercombination, and forbidden lines for increasing wavelength). Upper right panel shows best-fit (histogram) to RGS1 data (error bars) during quiescence, while upper left and lower left plots illustrate deviations for densities of $1.510^{10} \mathrm{~cm}^{-3}$ and for $4.510^{10} \mathrm{~cm}^{-3}$, corresponding to the $90 \%$ confidence limits of $n_{\mathrm{e}}$. The lower right panel shows the triplet for data selected during the two large flares

time-resolved spectral fitting of different sections of the largest flare $(0.9 \mathrm{~d})$, and of the quiescent emission. For the EPIC pn, a quiescent spectrum was extracted from the first (pre-flare) $18 \mathrm{ks}$ after the observing gap of the first observation, and fitted in the $0.15-10 \mathrm{keV}$ range with multi- $T$ VMEKAL components. We further chose five intervals across the flare, defined as I1-I5 henceforth (Fig. 2). They contain a roughly equal number of counts per spectrum (Table 4). All background-subtracted pn spectra were binned to a minimum of 20 counts per bin and fitted using XSPEC with the latest pn medium filter response matrix.

RGS2 spectra of both observations were fitted in SPEX. For quiescence, superior statistics was available from the second observation, although the best-fit results are very similar to the results from the shorter quiescent pieces in the first observation. Spectral analysis of the flare required stronger rebinning than for the pn in order to
Table 2. Time ranges of extracted pn spectra (in s from beginning of second part, after the gap, of first observation). Live exposure times for the data have been estimated as $66 \%$ of the observation time

\begin{tabular}{lrrrr}
\hline Interval & Start time & End time & Expos. time & Source counts \\
\hline Quiescent & 0 & 18050 & 11913 & 247790 \\
I1 & 22050 & 23111 & 700 & 25270 \\
I2 & 23111 & 23984 & 576 & 27780 \\
I3 & 23984 & 25111 & 744 & 27751 \\
I4 & 25111 & 26436 & 875 & 27685 \\
I5 & 26436 & 28050 & 1065 & 27946 \\
Bgnd & 0 & 35000 & 23100 & $\approx 9000$ \\
\hline
\end{tabular}

achieve a sufficient signal-to-noise ratio. We therefore fitted data from sections I2 and I3 together. 
Table 3. 3-temperature fit to the quiescent spectrum allowing individual elemental abundances ${ }^{\text {a }}$ to vary. The best-fitting values are given together with their $90 \%$ confidence intervals for a single parameter of interest $\left(\Delta \chi^{2}=2.71\right)$

\begin{tabular}{lllll}
\hline & \multicolumn{2}{c}{ pn (April 30/May 1): } & \multicolumn{2}{l}{ RGS2 } \\
Parameter & Value & $90 \%$ & Value & $90 \%$ \\
\hline$k T_{1}[\mathrm{keV}]$ & 0.11 & $(0.106-0.114)$ & 0.32 & $(0.296-0.337)$ \\
$k T_{2}[\mathrm{keV}]$ & 0.62 & $(0.609-0.627)$ & 0.68 & $(0.671-0.687)$ \\
$k T_{3}[\mathrm{keV}]$ & 1.90 & $(1.762-2.002)$ & 1.93 & $(1.79-2.14)$ \\
$E M_{1}\left[10^{51} \mathrm{~cm}^{-3}\right]$ & 16.1 & $(14.7-17.4)$ & 8.30 & $(7.10-9.54)$ \\
$E M_{2}\left[10^{51} \mathrm{~cm}^{-3}\right]$ & 57.3 & $(54.1-60.3)$ & 36.5 & $(34.0-39.2)$ \\
$E M_{3}\left[10^{51} \mathrm{~cm}^{-3}\right]$ & 19.6 & $(18.2-21.7)$ & 33.4 & $(31.5-35.2)$ \\
$\mathrm{C}$ & $=0.46$ & $\ldots$ & 0.46 & $(0.26-0.69)$ \\
$\mathrm{N}$ & $=0.53$ & $\ldots$ & 0.53 & $(0.46-0.61)$ \\
$\mathrm{O}$ & 0.49 & $(0.46-0.52)$ & 0.40 & $(0.37-0.43)$ \\
$\mathrm{Ne}$ & 0.70 & $(0.61-0.79)$ & 0.99 & $(0.92-1.06)$ \\
$\mathrm{Mg}$ & 0.31 & $(0.28-0.36)$ & 0.27 & $(0.21-0.33)$ \\
$\mathrm{Si}$ & 0.29 & $(0.26-0.33)$ & 0.14 & $(0.05-0.23)$ \\
$\mathrm{S}$ & 0.38 & $(0.29-0.48)$ & 0.04 & $(0.01-0.07)$ \\
$\mathrm{Ar}$ & 1.13 & $(0.79-1.46)$ & 0.86 & $(0.60-1.16)$ \\
$\mathrm{Ca}$ & $=0.18$ & $\ldots$ & 0.18 & $(0.00-0.43)$ \\
$\mathrm{Fe}$ & 0.19 & $(0.17-0.20)$ & 0.22 & $(0.21-0.23)$ \\
$\mathrm{Ni}$ & 1.33 & $(1.13-1.55)$ & 0.47 & $(0.36-0.57)$ \\
\hline
\end{tabular}

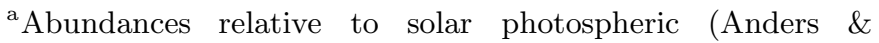
Grevesse 1989.)

\subsection{The quiescent spectrum}

The spectral fits of the EPIC pn spectrum used a common global abundance $Z$ and a fixed absorbing column of $210^{18} \mathrm{~cm}^{-2}$ (e.g., Rucinski et al. 1995). A 4-T MEKAL fit using solar photospheric abundances was found to be significantly better than a $3-T$ fit $\left(\chi^{2} / \nu=820 / 550\right.$ versus $\left.\chi^{2} / \nu=1077 / 552\right)$, but neither was formally acceptable and inspection of the residuals showed evidence for line emission suggesting abundance differences among metals (relative to solar values). The single-parameter $90 \%$ confidence interval for the global abundance was $(0.22-$ $0.25)$ solar. The spectrum was therefore fitted with a $3-T$ VMEKAL model that allowed abundance variations between elements. The abundances of C, N, and Ca were set to their RGS values since these elements show only very weak or unresolved features in the pn spectrum. This produced a much better fit $\left(\chi^{2} / \nu=696 / 543\right)$, detailed in Table 3 and illustrated in Fig. 6.

Table 3 also reports the RGS2 best-fit results of the second observation. There is acceptable agreement between pn and RGS, with a few exceptions. We note, however, that the instruments are complementary: The pn provides good results for high temperatures and the elements $\mathrm{Si}, \mathrm{S}$, Ar, and Fe, while the RGS is sensitive to intermediate temperatures and provides good access to strong, individual lines in particular of $\mathrm{C}, \mathrm{N}, \mathrm{O}, \mathrm{Ne}$, $\mathrm{Mg}$, Si, and Fe. The discrepancies for $\mathrm{S}$ and $\mathrm{Si}$ are most likely due to some poor atomic data used in the code, related to L-shell transitions of these elements (see Audard et al. 2001). Overall, the results show underabundances of all elements relative to solar photospheric values (Anders $\&$ Grevesse 1989). Note that elements with a high First Ionization Potential (FIP), such as C, N, O, and Ne, tend to show higher abundances than some low-FIP elements (Fe, Mg, Si). This "inverse FIP effect" was also noted by

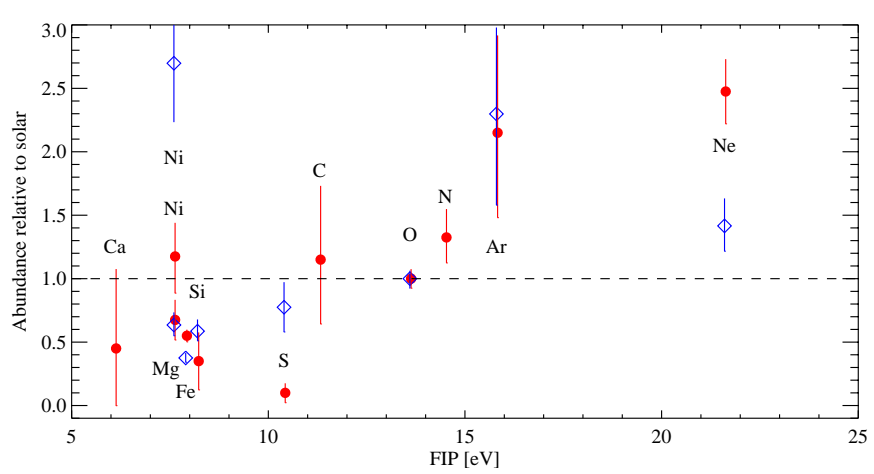

Fig. 5. Elemental abundances relative to solar photospheric values (Anders \& Grevesse 1989), normalized to the oxygen abundance. Filled circles (red): from RGS; diamonds (blue): from pn

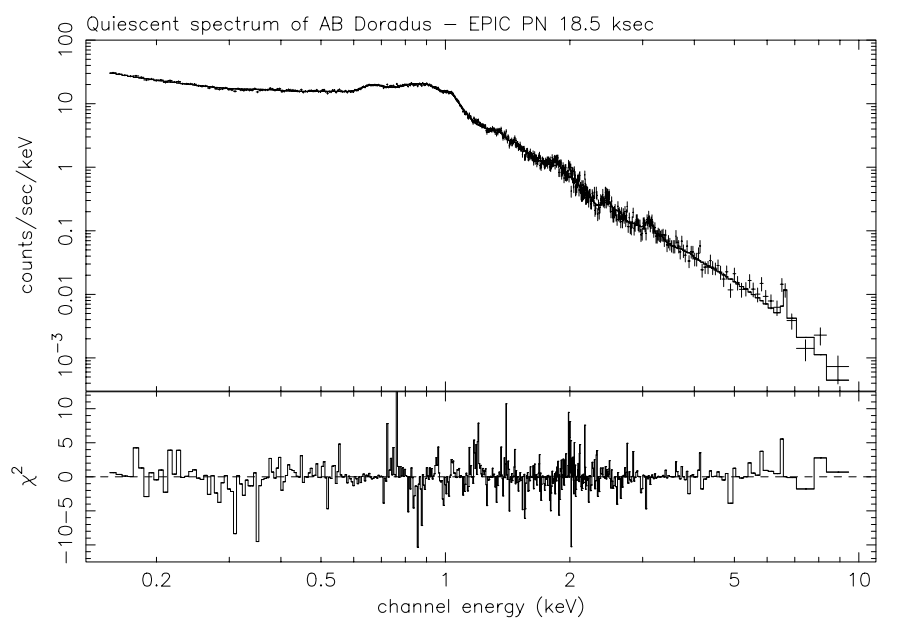

Fig. 6. 3-temperature fit to the EPIC pn quiescent spectrum. The $\chi^{2}$ residuals are also shown

Brinkman et al. (2001) for HR 1099. Abundances from RGS and pn (only RGS for C, N, and Ca) are illustrated in Fig. 5, normalized to the oxygen abundance. The abundances of $\mathrm{Ne}$ and $\mathrm{Ar}$ (the two highest-FIP elements) and $\mathrm{Ni}$ are particularly high (disagreement between pn and RGS results for $\mathrm{Ne}$ and $\mathrm{Ni}$, however). We note that the results are overall remarkably consistent with findings from $A S C A$ and EUVE spectroscopy (Mewe et al. 1996).

\subsection{The flare spectra}

The pn spectra of the flare at $0.9 \mathrm{~d}$ were modelled with the fixed 3-T quiescent model (Sect. 4.1) plus a single VMEKAL plasma component. Given the short timescales of the flare and the limited statistics, we fitted a "global metallicity" $Z$ instead of individual abundances. Note, however, that $Z$ is largely dominated by the element Fe. The results for each flare section can be seen in Table 4.

The emission measure of the flare component follows the overall flux level. The temperature and the global metallicity $Z$ appear to be highest during the rise phase of the flare (I1) and decrease thereafter, although the 
Table 4. 4-temperature fits to the flare section spectra. Three components are fixed to the quiescent model while the temperature, normalisation and global abundance $Z$ of the fourth component are allowed to vary. The final column shows the improvement in $\chi^{2}$ over a similar fit with the elemental abundances constrained to their individual quiescent levels. For RGS2, $k T_{4}$ was constrained to $\leq 2.5 \mathrm{keV}$

\begin{tabular}{|c|c|c|c|c|c|c|c|c|c|c|}
\hline Instrument & Interval & $k T_{4}$ & $90 \%$ & $E M_{4}^{\mathrm{a}}$ & $90 \%$ & $Z_{4}$ & $90 \%$ & $\chi^{2} / \nu$ & $\chi_{\nu}^{2}$ & $\Delta \chi^{2}$ \\
\hline \multirow[t]{5}{*}{$\overline{\mathrm{pn}:}$} & I1 & 2.85 & $\overline{(2.71-3.06)}$ & 31.7 & $(30.3-33.0)$ & 0.502 & $(0.402-0.610)$ & $454 / 390$ & 1.17 & 32 \\
\hline & $\mathrm{I} 2$ & 2.40 & $(2.19-2.51)$ & 51.4 & $(59.7-53.1)$ & 0.416 & $(0.353-0.483)$ & $524 / 396$ & 1.32 & 42 \\
\hline & I3 & 1.72 & $(1.65-1.79)$ & 32.1 & $(30.8-33.4)$ & 0.287 & $(0.243-0.336)$ & $482 / 369$ & 1.31 & 17 \\
\hline & $\mathrm{I} 4$ & 1.73 & $(1.64-1.82)$ & 21.6 & $(20.5-22.7)$ & 0.221 & $(0.170-0.279)$ & $438 / 354$ & 1.24 & 1 \\
\hline & I5 & 1.96 & $(1.75-2.14)$ & 11.0 & $(10.1-11.9)$ & 0.183 & $(0.088-0.292)$ & $351 / 340$ & 1.03 & 0 \\
\hline RGS2: & I2\&I3 & 2.5 & $(1.12-2.5)$ & 106 & $(85.1-126.3)$ & 0.349 & $(0.127-0.642)$ & $60 / 242$ & 0.25 & -1.8 \\
\hline
\end{tabular}

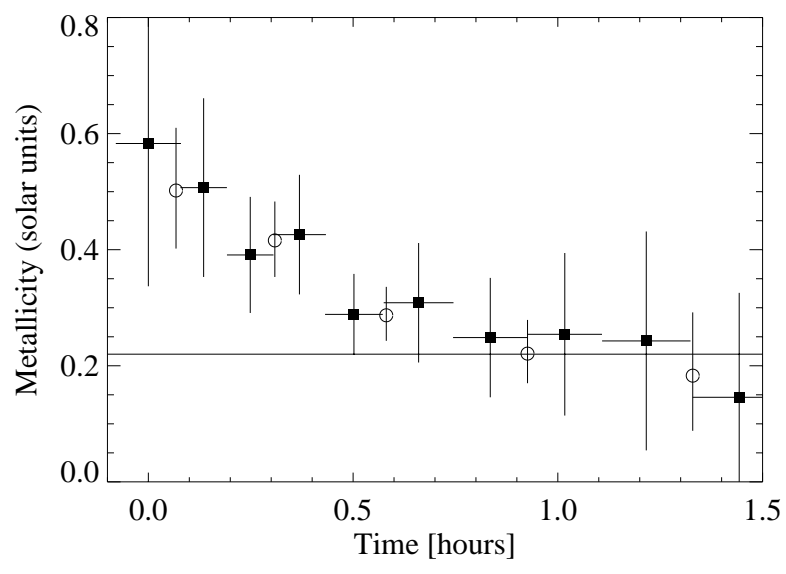

Fig. 7. Time variation of the best-fit plasma metallicity $Z$ during the flare. The open symbols refer to the results reported in Table 4; the filled circles show $Z$ at double resolution. Only the time intervals (horizontal error bars) related to the latter results are shown, for clarity reasons

temperature seems to stall as it falls to the temperature of the hottest quiescent component. Only in the final two sections is the metallicity statistically consistent with the quiescent $Z$ value (Sect. 4.1). We have repeated the metallicity analysis at higher time resolution, and all results are summarized in Fig. 7. This plot confirms that an abundance enhancement by a factor $\approx 3$ occurred at the very beginning of the flare. The results for RGS2 (combined intervals I2 and I3) are also reported in Table 4 . The bestfit $Z$ abundance is similar to the pn value, although with large confidence error ranges.

\section{Flare decay analysis}

We have analyzed the decay phase of the flare at $0.9 \mathrm{~d}$ with the approach developed by Reale et al. (1997) and Reale \& Micela (1998) in order to derive the dimension of the flaring stellar loops. We refer to these references for further information. The method is based on detailed hydrodynamic models, and it is sensitive to the presence of sustained heating during the flare decay. For the present study, the technique has been calibrated (courtesy of F. Reale) for the EPIC pn response, in the energy band 0.15$10 \mathrm{keV}$.

We divided the whole flare into 10 time bins, by splitting in two each interval reported in Table 4 in order to get an adequate sampling of the flare decay. Spectral analysis was performed as in Sect. 4.2. The flare decay shows a double-exponential behavior (Fig. 8a) with an initial e-folding decay time, $\tau_{\mathrm{LC}}$, of about one hour. We first determine the slope $\zeta$ of the decay in the $\log T$ vs. $\log \sqrt{E M}$ diagram (Fig. 8b). The method is applicable in the range $0.4<\zeta \leq 1.9$, where the lower bound corresponds to the case of a flare decay entirely driven by sustained heating, and the upper bound to the case of no heating during the decay. We find a best-fit slope $0.8<\zeta<1.1$. This result thus implies continuous loop heating, corresponding to a heating decay time of $\approx 2 \mathrm{ks}$. The resulting loop semilength is $L=(2.5 \pm 1.5) 10^{10} \mathrm{~cm}$, which implies for a loop in hydrostatic equilibrium - a plasma pressure of $\approx 510^{3}$ dyne $\mathrm{cm}^{-2}$, at the time of the $T$ peak (loop apex $T_{\max }=710^{7} \mathrm{~K}$ ), and a maximum heating rate of $\approx 4 \mathrm{erg} \mathrm{cm}^{-3} \mathrm{~s}^{-1}$ (Rosner et al. 1978).

\section{Conclusions}

Our observations present $\mathrm{AB}$ Dor in many facets: Quiescent, slowly varying, and moderately flaring intervals have been investigated spectroscopically, using both CCD and grating spectra from XMM-Newton. Spectroscopy of the He-like $\mathrm{O}$ vi lines allows us to measure an average coronal density of $310^{10} \mathrm{~cm}^{-3}$ for plasma of $\approx 1-3 \mathrm{MK}$. No change is seen during the flares. We attribute this result to the fact that flare plasma is predominantly hot and does not significantly contribute to the O viI line flux.

All data sets show low elemental abundances (below solar photospheric) although the photospheric abundances of AB Dor are near-solar (Vilhu et al. 1987). This result confirms previous measurements with low-resolution devices, e.g. onboard $A S C A$ and $E U V E$ (Mewe et al. 1996; Ortolani et al. 1998) and BeppoSAX (Maggio et al. 2000). Interestingly, the high-FIP elements, in particular Ne, show very high relative abundances. There is, in fact, evidence for an "inverse FIP effect", i.e., abundances increase 

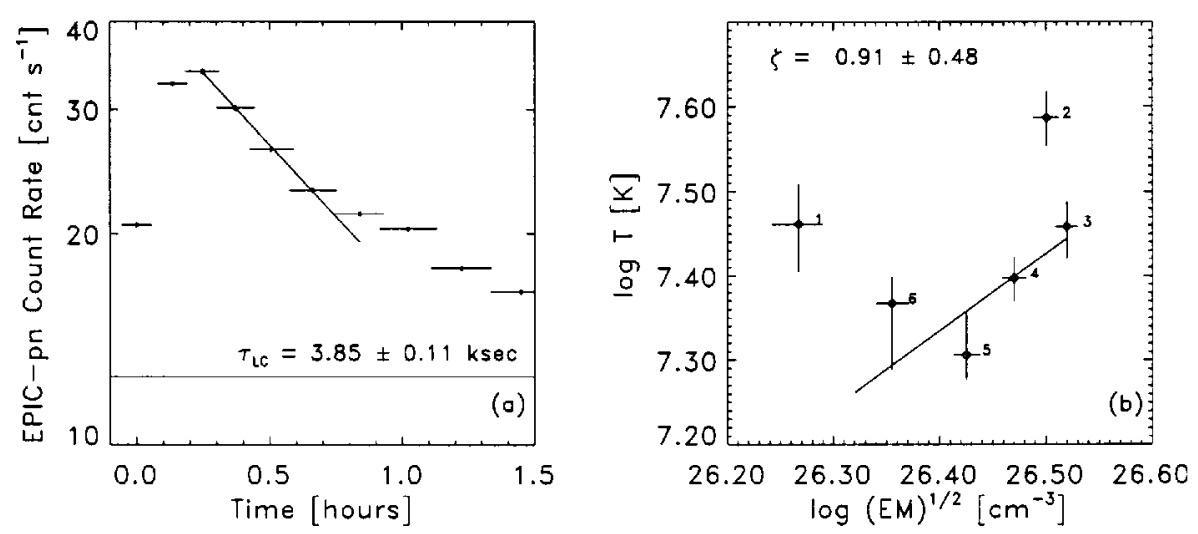

Fig. 8. a) EPIC pn light curve with best-fit exponential law. The horizontal line indicates the quiescent emission level. b) Flare evolution in the $\log T$ vs. $\log \sqrt{E M}$ diagram with best-fit linear regression curve (for points $3-6$ ). The numbers indicate the first 6 time intervals in a)

with increasing FIP, contrary to solar coronal behavior (Meyer 1985; Feldman et al. 1992). A detailed study of the inverse FIP effect is presented by Brinkman et al. (2001), who used the XMM-Newton RGS instruments for a deep exposure of the X-ray emission of HR 1099. During the larger flare at $0.9 \mathrm{~d}$, we detect an increase of the metal abundance (dominated by $\mathrm{Fe}$ ), which however rapidly decays back (within $\approx 30 \mathrm{~min}$ ) to the quiescent level. At the same time, the temperature of the flare component decreases, but only to a value corresponding to the hotter component in the quiescent emission. It appears that the plasma in these flaring loops is maintained at high temperatures long after the flare peak.

This flare has been modelled with the same approach used recently in the interpretation of a number of $\mathrm{X}$ ray flares seen from $\mathrm{AB}$ Dor with $A S C A$ and BeppoSAX (Ortolani et al. 1998; Maggio et al. 2000). Our analysis suggests continuous heating during the decay on a time scale of $\approx 2 \mathrm{ks}$ and a loop semilength of $\approx 2.5 \quad 10^{10} \mathrm{~cm}$. Assuming for $\mathrm{AB}$ Dor a radius of $\approx 1 R_{\odot}$ (Maggio et al. 2000 ), the loop semilength is $\approx 0.3 R_{\star}$. This is similar to or somewhat smaller than the sizes derived with the same method for two large flares on AB Dor observed by BeppoSAX (which gave loop semilengths of $3.510^{10}$ and $4.910^{10} \mathrm{~cm}$, respectively; Maggio et al. 2000) and for a moderate-size flare seen by $A S C A$ (which resulted in a loop semilength of $3.210^{10} \mathrm{~cm}$; Ortolani et al. 1998). Note that the flare seen by $X M M-N e w t o n$, with an estimated total energy of $\approx 10^{34} \mathrm{erg}$, is much smaller than the flares seen by BeppoSAX which had a total energy in X-rays two orders of magnitude larger and reached a much higher coronal temperature $\left(\approx 10^{8} \mathrm{~K}\right)$. This indicates the extreme variability of the magnetically active corona of AB Dor, ranging from relatively quiescent phases (as observed during the XMM-Newton observation on 2000 June 7) to moderate-sized flares, and to episodes of extremely powerful flares. Yet, the typical dimensions of all these flares derived with the same method do not differ by more than a factor of 2 and represent a significant fraction of the stellar radius.
Acknowledgements. We thank the referee for constructive comments on the paper. We also acknowledge the help provided by F. Reale who performed the calibration of the flare decay analysis method with the EPIC-pn response. M. A. acknowledges support from the Swiss National Science Foundation (grants 2100-049343 and 2000-058827), from the Swiss Academy of Sciences, and from the Swiss Commission for Space Research. H. M., K. B, and J. P. acknowledge financial support from PPARC. A. M. and R. P. acknowledge support from the Italian Space Agency. SRON is supported financially by NWO.

\section{References}

Anders, E., \& Grevesse, N. 1989, Geochim. Cosmochim. Acta, 53, 197

Arnaud, K. A. 1996, in ASP Conf. Ser. 101, Astronomical Data Analysis Software and Systems V, ed. G. Jacoby, \& J. Barnes (San Francisco: ASP), 17

Audard, M., Behar, E., Güdel, M., et al. 2001, A\&A, 365, L329

Brinkman, A. C., Behar E., Güdel M., et al. 2001, A\&A, 365, L324

Collier Cameron, A., \& Foing, B. H. 1997, Observatory, 117, 218

Collier Cameron, A., Bedford, D. K., Rucinski, S. M., Vilhu, O., \& White N. E. 1988, MNRAS, 231, 131

ESA 1997, in European Space Agency SP-1200

Feldman, U., Mandelbaum, P., Seely, J. F., Doschek, G. A., \& Gursky, H. 1992, ApJS, 81, 387

Guirado, J. C., Reynolds, J. E., Lestrade, J.-F., et al. 1997, ApJ, 490, 835

den Herder, J. W., Brinkman, A. C., Kahn, S. M., et al. 2001, A\&A, 365, L7

Innis, J. L., Thompson, K., Coates, D. W., \& Lloyd Evans, T. 1998, MNRAS, 235, 1422

Jansen, F., Lumb, D., Altieri, B., et al. 2001, A\&A, 365, L1

Kaastra, J. S., Mewe, R., \& Nieuwenhuijzen, H. 1996, in UV and X-ray Spectroscopy of Astrophysical and Laboratory Plasmas, ed. K. Yamashita, \& T. Watanabe (Tokyo: Universal Academy), 411

Kürster, M., Schmitt, J. H. M. M., Cutispoto, G., \& Dennerl, K. 1997, A\&A, 320, 831

Maggio, A., Pallavicini, R., Reale, F., \& Tagliaferri, G. 2000, A\&A, 356, 627 
Mewe, R., Kaastra, J. S., White, S. M., \& Pallavicini, R. 1996, A\&A, 315, 170

Mewe, R., Porquet, D., Raassen, A. J. J., \& Dubau, J. 2001, in preparation

Meyer, J.-P. 1985, ApJS, 57, 173

Ortolani, A., Pallavicini, R., Maggio, A., Reale, F., \& White, S. M. 1998, in 10th Cambridge Workshop on Cool Stars, Stellar Systems, and the Sun, ed. R. A. Donahue, \& J. A. Bookbinder (San Francisco: ASP), 1532

Pakull, M. W. 1981, A\&A, 104, 33

Reale, F., \& Micela, G. 1998, A\&A, 334, 1028

Reale, F., Betta, R., Peres, G., Serio, S., \& McTiernan, J. 1997, A\&A, 325, 782

Rosner, R., Tucker, W. H., \& Vaiana, G. S. 1978, ApJ, 220, 643
Rucinski, S. M., Mewe, R., Kaastra, J. S., Vilhu, O., \& White, S. M. 1995, ApJ, 449, 900

Strüder, L., Briel, U. G., Dennerl, K., et al. 2001, A\&A, 365, L18

Turner, M. J. L., Abbey, A., Arnaud, M., et al. 2001, A\&A, $365, \mathrm{~L} 27$

Vilhu, O., \& Linsky, J. L. 1987, PASP, 99, 1071

Vilhu, O., Gustafsson, B., Edvardsson, B., et al. 1987, ApJ, 320,850

Vilhu, O., Tsuru, T., Collier Cameron, A., et al. 1993, A\&A, 278,467

White, S. M., Pallavicini, R., \& Lim, J. 1996, in 9th Cambridge Workshop on Cool Stars, Stellar Systems, and the Sun, ed. R. Pallavicini, \& A. K. Dupree (San Francisco: ASP), 299 